\title{
Comparative analysis of the microbial communities in raw milk produced in different regions of Korea
}

\author{
In Seon Kim, Yoo Kyung Hur ${ }^{2}$, Eun Ji Kim², Young-Tae Ahn ${ }^{3}$, Jong Geun Kim², \\ Yun-Jaie Choi ${ }^{1,5, *}$, and Chul Sung Huh ${ }^{2,4, *}$
}

\footnotetext{
* Corresponding Authors: Yun-Jaie Choi Tel: +82-2-880-4807, Fax: +82-2-880-7340, E-mail: cyjcow@snu.ac.kr

Chul Sung Huh

Tel: +82-33-339-5723, Fax: +82-33-339-5855

E-mail: cshuh@snu.ac.kr

'Department of Agricultural Biotechnology, Seoul National University, Seoul 08826, Korea

${ }^{2}$ Graduate School of International Agricultural

Technology, Seoul National University, Pyeongchang 25354, Korea

${ }^{3}$ R\&BD Center, Korea Yakult Co., Ltd., Yongin 17086, Korea

${ }^{4}$ Institute of Green-Bio Science \& Technology, Seoul National University, Pyeongchang 25354, Korea

${ }^{5}$ Research Institute for Agriculture and Life Science,

Seoul National University, Seoul 08826, Korea
}

Submitted Jul 17, 2017; Revised Sept 18, 2017; Accepted Sept 19, 2017
Objective: The control of psychrotrophic bacteria causing milk spoilage and illness due to toxic compounds is an important issue in the dairy industry. In South Korea, Gangwon-do province is one of the coldest terrains in which eighty percent of the area is mountainous regions, and mainly plays an important role in the agriculture and dairy industries. The purposes of this study were to analyze the indigenous microbiota of raw milk in Gangwon-do and accurately investigate a putative microbial group causing deterioration in milk quality.

Methods: We collected raw milk from the bulk tank of 18 dairy farms in the Hoengseong and Pyeongchang regions of Gangwon-do. Milk components were analyzed and the number of viable bacteria was confirmed. The V3 and V4 regions of 16S rRNA gene were amplified and sequenced on an Illumina Miseq platform. Sequences were then assigned to operational taxonomic units, followed by the selection of representative sequences using the QIIME software package.

Results: The milk samples from Pyeongchang were higher in fat, protein, lactose, total solid, and solid non-fat, and bacterial cell counts were observed only for the Hoengseong samples. The phylum Proteobacteria was detected most frequently in both the Hoengseong and Pyeongchang samples, followed by the phyla Firmicutes and Actinobacteria. Notably, Corynebacterium, Pediococcus, Macrococcus, and Acinetobacter were significantly different from two regions. Conclusion: Although the predominant phylum in raw milk is same, the abundances of major genera in milk samples were different between Hoengseong and Pyeongchang. We assumed that these differences are caused by regional dissimilar farming environments such as soil, forage, and dairy farming equipment so that the quality of milk raw milk from Pyeongchang is higher than that of Hoengseong. These results could provide the crucial information for identifying the microbiota in raw milk of South Korea.

Keywords: Raw Milk; Microbiota; Milk Quality; Psychrotrophic Bacteria

\section{INTRODUCTION}

Raw milk is a highly nutritious food that contains proteins, fats, carbohydrates, vitamins, minerals, and essential amino acids, and has a near neutral $\mathrm{pH}$ and high water activity. Therefore, milk can also provide ideal conditions for the growth of many microorganisms [1]. The raw milk microbiota is usually introduced from a variety of sources including the teat surface, air, water, soil, feed, grass, milking equipment, and other sources [2].

Some microorganisms in raw milk, including members of the bacterial genera Lactococcus, Lactobacillus, Streptococcus, Propionibacterium as well as fungi can directly impact the sensory, texture, flavor, and organoleptic properties of dairy products [3]. Moreover, Lactobacillus and Bifidobacterium can promote human health by digesting certain nutrients or by reducing allergies such as asthma and atopic diseases [4]. However, other microorganisms can also negatively im- 
pact milk quality and human health. The main cause of illness from the consumption of milk products is the contamination with Listeria spp., Salmonella spp., Escherichia coli, Campylobacter spp., Shigella spp., and mycotoxin-producing fungi [2]. These pathogenic microorganisms can lead to fever, nausea, vomiting, diarrhea, and abdominal pains.

In addition, the psychrotrophic bacteria have the ability to grow at low temperatures and can be a major cause of milk spoilage, persisting and proliferating during cold storage and producing lipases and proteases [5]. The cold-resistant bacteria in milk are predominated by Gram-negative genera (Pseudomonas, Achromobacter, Aeromonas, Serratia, Alcaligenes, Chromobacterium, and Flavobacterium spp.), and the lower numbers of Gram-positive genera (Bacillus, Clostridium, Corynebacterium, Streptococcus, Lactobacillus, and Microbacterium spp.) are usually distributed in milk [6]. Due to enzymatic and microbial activity, the milk quality is highly related to the microbial community in raw milk. Thus, the incidence of psychrotrophic bacteria mainly causes the deterioration of dairy products. Microbial proteases can reduce the nutrient and economic value of milk by the hydrolysis of casein during the milk processes. Additionally, lipases which is byproduct of microbial activity, convert the lipids into free fatty acids and partial glycerides. The increase of free fatty acids in milk can cause unexpected flavor change and altered organoleptic properties [7]. Although the microbial quality of milk has been improved significantly through the cooling and tank lorry systems, it is still difficult for many dairy producers to control the milk microbiota. The inhibition of cold-tolerant microorganisms in milk is a very important task in dairy industry. However, the distribution of specific raw microorganisms in South Korea has not been studied yet.

In South Korea, an annual average production of raw milk is 2,168,000 tons from approximately 5,498 dairy farms and 197,105 milking cows [8]. Gangwon-do province occupies an area of $20,569 \mathrm{~km}^{2}$ and $4 / 5$ of which is a mountainous forest area. In Gangwon-do, Hoengseong and Pyeongchang counties mainly play an important role in agriculture and dairy industry. Thus, in this study, we compared the milk contents and core microbiota between the Hoengseong and Pyeongchang samples to confirm the core microbiota in raw milk derived from the geographical differences in Gangwon-do.

\section{MATERIALS AND METHODS}

\section{Farm selection and raw milk sampling}

To characterize the indigenous microbiota of raw milk from the Hoengseong and Pyeongchang counties, 9 representative farms that produce approximately $1,500 \mathrm{~kg} / \mathrm{d}$ of milk were selected. The herd size of the farms ranged from 20 to 100, with the average of the Hoengseong and Pyeongchang groups being similar. Every farm stores milk refrigerated below $4^{\circ} \mathrm{C}$ in bulk tanks. Information regarding the farms is presented in Supplementary Table
S1. Raw milk was collected with a sterile $500 \mathrm{~mL}$ bottle from the bulk tanks of the selected farms. Samples were transported on ice and kept at $-20^{\circ} \mathrm{C}$ until experiments were performed.

Raw milk components analysis and viable bacteria counting Milk components were analyzed using a MilkoScan 6,000 series (Foss Electric Co, Hillerd, Denmark), assaying for lactose, protein, fat, total solid (TS), and solid non-fat (SNF) content. Microbiological enumeration was performed by spreading aliquots of serially diluted milk aliquot onto agar plates. The number of total viable bacteria was determined by standard plate count, cultured aerobically at $30^{\circ} \mathrm{C}$ for 48 hour [9] and psychrotrophic bacterial counts were determined by incubating plates for 10 days at $7^{\circ} \mathrm{C}$ [10]. Counts of lactic acid bacteria were determined by MRS (Man, Rogosa, and Sharpe) at $30^{\circ} \mathrm{C}$ for 48 hour. Bacterial numbers were counted using plates containing between 30 and 300 colonies.

\section{Bacterial genomic DNA extraction}

To collect bacterial cells from samples, aliquots $(20 \mathrm{~mL})$ of raw milk were centrifuged at $14,000 \times g$ for $30 \mathrm{~min}$ at $4^{\circ} \mathrm{C}$. Fat and supernatants were removed and the pellet was resuspended in 20 $\mathrm{mL}$ of phosphate-buffered saline $(\mathrm{pH}=7.2 ; 137 \mathrm{mM}$ of NaCl, 2.68 $\mathrm{mM}$ of $\mathrm{KCl}, 10.1 \mathrm{mM}$ of $\mathrm{Na}_{2} \mathrm{HPO}_{4}, 1.76 \mathrm{mM}$ of $\mathrm{KH}_{2} \mathrm{PO}_{4}$ ) [11]. The mixture was then centrifuged again for $30 \mathrm{~min}$ at $14,000 \times \mathrm{g}$, $4^{\circ} \mathrm{C}$ to remove residual fat. The resulting pellet was used to isolate genomic DNA using a FastDNA SPIN Kit for Soil (MP Bio, Solon, $\mathrm{OH}, \mathrm{USA}$ ) according to the manufacturer's instructions with some modification. The purity and integrity of the extracted DNA were measured by a SpectraMax M3 (Molecular device, Sunnyvale, CA, USA) microplate reader.

High-throughput sequencing and bioinformatics analysis The V3 and V4 regions of the 16S rRNA gene were amplified with primer pair that creates a single amplicon of approximately $460 \mathrm{bp}$, including sequence for compatibility with Illumina index and sequencing adapters. The polymerase chain reaction (PCR) products were purified using a QIAquick PCR purification kit (Qiagen, Hilden, Germany) and sequenced on an Illumina Miseq platform by a commercial company (Macrogen Inc., Seoul, South Korea). Sequencing data were converted into raw data for the analysis. To analyze the $16 \mathrm{~S}$ rRNA sequences, quality filtering, trimming and error free read identification steps were performed. The Phred quality (Q score) was used to evaluate the read qualities. Sequences were then assigned to operational taxonomic units (97\% identity), followed by the selection of representative sequences using the QIIME software package [12]. Bacterial clustering was analyzed at the phylum and genus levels, and to reveal generated rarefaction curves, Shannon-Wiener and Chaol diversity indices as well as and Good's coverage estimator values were used. In addition, we performed a UniFrac analysis and a principal coordinate analysis ( $\mathrm{PCoA}$ ). The distances between microbial communities from each sample were represented as an unweighted 
pair-group method with arithmetic mean (UPGMA) clustering between multiple samples.

\section{Statistical analysis}

All data were statistically analyzed using Graph Pad Prism software Version 5.01 (Graph Pad Software, San Diego, CA, USA). The statistical significance of the differences was determined by one-way analysis of variance (ANOVA) followed by Tukey's post hoc test or two-way ANOVA followed by Bonferroni post-tests. Differences were considered significant at $\mathrm{p}<0.05$.

\section{RESULTS}

Raw milk composition and bacterial cell counts

To analyze the composition and population of microbiota in raw milk between the Hoengseong and Pyeongchang counties of Gangwon-do province, we collected raw milk from the bulk tanks of 18 farms, 9 from Hoengseong and 9 from Pyeongchang, respectively. All samples contained $2.72 \%$ to $4.09 \%$ of fat, $2.90 \%$ to $3.23 \%$ of protein, $4.44 \%$ to $4.65 \%$ of lactose and $8.41 \%$ to $8.93 \%$ of SNF (Table 1). The contents of fat, protein, and lactose were slightly higher in raw milk from Pyeongchang than from Hoengseong, although this was not statistically significant in both datasets. Significant differences $(p<0.05)$ were found for the TS values in raw milk. Total viable cell counts at $30^{\circ} \mathrm{C}$ were in the range of
2.85 to $4.78 \log _{10} \mathrm{cfu} / \mathrm{mL}$. In addition, the viability of psychrotrophic bacteria within each sample was detected in the range of 1.60 to $3.00 \log _{10} \mathrm{cfu} / \mathrm{mL}$. These results demonstrate that the composition and viable bacterial cell numbers in raw milk from Pyeongchang were higher than from Hoengseong.

Quality control and diversity analysis of sequencing data The number of clean reads, Q30 quality scores, diversity, and richness estimators for each sample and group are shown in Table 2. The number of reads after ambiguous, low quality and chimera sequences were removed varied from 15,203 to 61,364 . A total of 788,513 reads were classified as bacteria and were used to calculate relative abundances. The Q30, and Shannon and Chao1 diversity indices were not significantly different. Good's coverage, which was used to evaluate the sequencing depth, is represented by a value near 1. All samples had Good's coverage values between 0.99915 and 0.99995 . Rarefaction analysis demonstrated that the sequencing depth of all samples was sufficient and that the sample diversity of Hoengseong was slightly higher than from Pyeongchang (Supplementary Figure S1).

\section{Relative abundances of raw milk microbiota}

The relative microbial abundances in raw milk from Hoengseong and Pyeongchang are reported at both the phylum and genus levels (Figure 1). Furthermore, the average percentage of the

Table 1. The milk composition and the number of total viable psychrotrophic bacteria in raw milk samples from Hoengseong and Pyeongchang ${ }^{1)}$

\begin{tabular}{|c|c|c|c|c|c|c|c|}
\hline Sample & Fat (\%) & Protein (\%) & Lactose $(\%)$ & TS (\%) & SNF (\%) & $\begin{array}{l}\text { Total bacterial } \\
\text { count at PCA } \\
\left(\log _{10} \text { cfu/mL) }\right.\end{array}$ & $\begin{array}{c}\text { Psychrotrophic } \\
\text { bacterial count } \\
\left(\log _{10} \mathrm{cfu} / \mathrm{mL}\right)\end{array}$ \\
\hline \multicolumn{8}{|l|}{ Hoengseong } \\
\hline 01 & 3.28 & 2.91 & 4.52 & 11.63 & 8.47 & 4.00 & 3.00 \\
\hline 02 & 3.22 & 3.15 & 4.58 & 11.88 & 8.73 & 3.30 & 1.90 \\
\hline 03 & 3.3 & 3.12 & 4.56 & 12.08 & 8.82 & 2.85 & 2.60 \\
\hline 04 & 2.95 & 2.9 & 4.45 & 11.38 & 8.49 & 4.78 & 3.30 \\
\hline 05 & 3.68 & 3.13 & 4.52 & 12.31 & 8.7 & 3.30 & 2.30 \\
\hline 06 & 3.17 & 2.81 & 4.46 & 11.54 & 8.41 & 3.78 & 2.90 \\
\hline 07 & 3.97 & 3.23 & 4.44 & 12.53 & 8.65 & 2.95 & 3.70 \\
\hline 09 & 4.09 & 3.23 & 4.46 & 12.7 & 8.7 & 4.00 & 2.30 \\
\hline 10 & 2.72 & 3.03 & 4.55 & 11.36 & 8.7 & 2.85 & 2.78 \\
\hline Mean \pm SD & $3.38 \pm 0.45$ & $3.06 \pm 0.15$ & $4.50 \pm 0.05$ & $11.93 \pm 0.50^{\mathrm{a}}$ & $8.63 \pm 0.14$ & $3.53 \pm 0.66$ & $2.75 \pm 0.55$ \\
\hline \multicolumn{8}{|c|}{ Pyeongchang } \\
\hline 11 & 3.12 & 3.11 & 4.53 & 11.79 & 8.69 & 3.48 & 2.48 \\
\hline 12 & 3.06 & 3.16 & 4.56 & 11.85 & 8.80 & 2.95 & 1.60 \\
\hline 13 & 3.41 & 2.98 & 4.61 & 12.02 & 8.64 & 3.48 & 2.70 \\
\hline 14 & 4.05 & 3.11 & 4.55 & 12.70 & 8.74 & 3.00 & 1.90 \\
\hline 15 & 3.57 & 3.15 & 4.54 & 12.33 & 8.79 & 2.95 & 2.30 \\
\hline 16 & 3.78 & 3.22 & 4.65 & 12.67 & 8.93 & 3.60 & 2.85 \\
\hline 17 & 3.66 & 3.06 & 4.53 & 12.24 & 8.61 & 4.48 & 2.48 \\
\hline 18 & 3.51 & 2.96 & 4.52 & 12.19 & 8.66 & 3.60 & 2.00 \\
\hline 19 & 3.74 & 3.14 & 4.59 & 12.44 & 8.76 & 4.48 & 3.00 \\
\hline Mean \pm SD & $3.54 \pm 0.32$ & $3.10 \pm 0.09$ & $4.56 \pm 0.04$ & $12.25 \pm 0.33^{b}$ & $8.74 \pm 0.10$ & $3.56 \pm 0.59$ & $2.37 \pm 0.46$ \\
\hline
\end{tabular}

TS, total solid; SNF, solid non-fat; PCA, plate count agar; SD, standard deviation

1) Two-way analysis of variance, Bonferroni post-tests.

a,b Means within a row with different superscripts differ significantly $(p<0.05)$. 
Table 2. The number of analyzed sequences, estimated quality score, OTU richness (Chao1), diversity (Shannon and Simpson) estimators, and sample coverage

\begin{tabular}{|c|c|c|c|c|c|c|}
\hline Sample & Clean reads & Q30 (\%) & Chao1 & Shannon & Simpson & Good's coverage \\
\hline \multicolumn{7}{|l|}{ Hoengseong } \\
\hline 01 & 55,551 & 87.01 & 335.75 & 7.31 & 0.99 & 0.99995 \\
\hline 02 & 60,118 & 87.90 & 481.50 & 7.49 & 0.98 & 0.99990 \\
\hline 03 & 58,991 & 87.13 & 329.00 & 7.48 & 0.99 & 0.99995 \\
\hline 04 & 61,364 & 87.62 & 356.50 & 6.36 & 0.95 & 0.99992 \\
\hline 05 & 59,792 & 87.40 & 367.67 & 6.16 & 0.92 & 0.99990 \\
\hline 06 & 43,638 & 87.24 & 428.00 & 7.66 & 0.99 & 0.99975 \\
\hline 07 & 51,362 & 87.92 & 392.75 & 7.59 & 0.99 & 0.99988 \\
\hline 09 & 56,159 & 87.58 & 335.00 & 7.36 & 0.99 & 0.99989 \\
\hline 10 & 41,910 & 87.34 & 429.50 & 7.68 & 0.99 & 0.99986 \\
\hline Mean \pm SD & $54,321 \pm 7,217$ & $87.46 \pm 0.32$ & $383.96 \pm 52.87$ & $7.23 \pm 0.57$ & $0.97 \pm 0.03$ & $0.99989 \pm 0.0001$ \\
\hline \multicolumn{7}{|l|}{ Pyeongchang } \\
\hline 11 & 15,203 & 87.28 & 330.00 & 6.90 & 0.97 & 0.99915 \\
\hline 12 & 29,428 & 85.87 & 771.33 & 7.52 & 0.97 & 0.99915 \\
\hline 13 & 32,436 & 87.63 & 369.00 & 7.57 & 0.99 & 0.99951 \\
\hline 14 & 38,011 & 87.28 & 511.55 & 7.67 & 0.98 & 0.99979 \\
\hline 15 & 29,700 & 87.53 & 376.14 & 7.61 & 0.99 & 0.99970 \\
\hline 16 & 32,250 & 87.72 & 392.63 & 7.10 & 0.98 & 0.99969 \\
\hline 17 & 31,293 & 87.88 & 368.11 & 6.63 & 0.96 & 0.99984 \\
\hline 18 & 37,695 & 87.43 & 382.50 & 7.29 & 0.99 & 0.99950 \\
\hline 19 & 53,612 & 88.00 & 429.75 & 4.81 & 0.91 & 0.99976 \\
\hline Mean \pm SD & $33,292 \pm 10,095$ & $87.40 \pm 0.63$ & $436.78 \pm 135.48$ & $7.01 \pm 0.90$ & $0.97 \pm 0.02$ & $0.99957 \pm 0.0003$ \\
\hline Total mean \pm SD & $43,806 \pm 13,767$ & $87.43 \pm 0.48$ & $410.37 \pm 103.40$ & $7.12 \pm 0.74$ & $0.97 \pm 0.024$ & $0.99970 \pm 0.0002$ \\
\hline
\end{tabular}

OTU, operational taxonomic units; SD, standard deviation.

microbial population that were greater than $0.5 \%$ of the total composition are presented at both the phylum (Table 3) and genus (Table 4) levels. In Hoengseong and Pyeongchang, the phylum Proteobacteria predominated, followed by Firmicutes and Actinobacteria. The abundance of Proteobacteria in the raw milk from Pyeongchang (31.98\%) was significantly higher than milk that originated from Hoengseong $(25.85 \%)$, whereas the Firmicutes population (18.07\%) was significantly lower than the Hoengseong groups (24.13\%). Several identifiable phyla in the Hoengseong and Pyeongchang groups were also detected, including Bacteroidetes (6.24\% and 6.99\%), Acidobacteria (4.58\% and 5.05\%), Planctomycetes (3.04\% and 3.20\%), Verrucomicrobia (2.96\% and 3.06\%), Chloroflexi (2.27\% and 2.10\%), Cyanobacteria (1.03\% and $0.60 \%)$, and Nitrospirae ( $0.67 \%$ and $0.66 \%)$, respectively. These bacterial abundances were significantly different between the groups.

At the genus level, the core microbiome of raw milk originating from Hoengseong and Pyeongchang encompassed members of Massilia (6.66\% and 9.17\%), Bacillus (5.14\% and 5.29\%), Corynebacterium (4.02\% and 1.47\%), Macrococcus (2.48\% and $1.18 \%$ ), Staphylococcus (1.92\% and 2.61\%), Arthrobacter (1.75\% and 2.02\%), Streptococcus (1.71\% and 2.52\%), and Burkholderia (1.66\% (a)

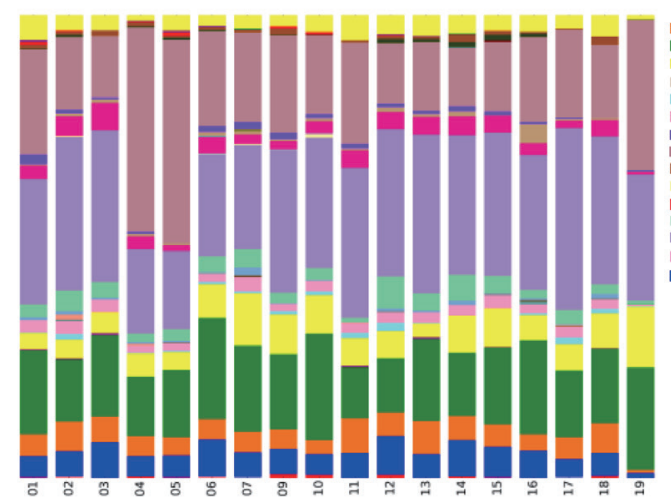

(b)

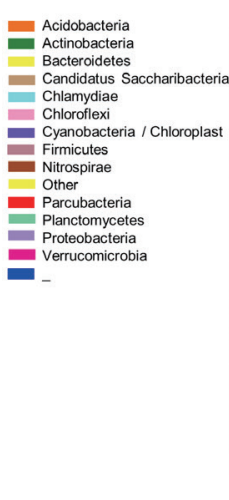

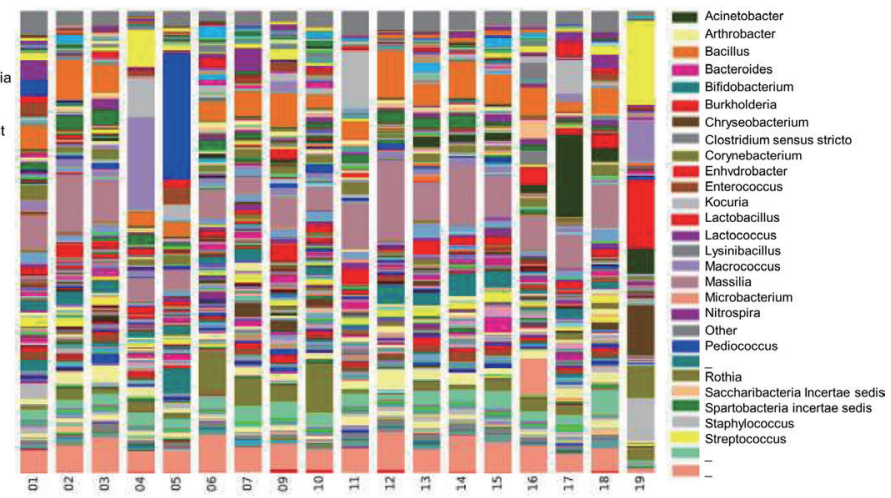

Figure 1. Relative abundances at the phylum (a) and genus (b) level within the bacterial community of raw milk samples from Hoengseong and Pyeongchang counties. The abundance was determined with partial $16 \mathrm{~S}$ rRNA genes sequences from bacteria in 18 raw milk samples. 
Table 3. The average percentage of predominant bacteria at the phylum level between the Hoengseong and Pyeongchang groups

\begin{tabular}{lccc}
\hline \multirow{2}{*}{ Phylum level } & \multicolumn{2}{c}{ Relative count (\%) } & \multirow{2}{*}{ p value } \\
\cline { 2 - 3 } & Hoengseong & Pyeongchang & \\
\hline Proteobacteria & $25.85 \pm 6.12$ & $31.98 \pm 3.46$ & $<0.001$ \\
Firmicutes & $24.13 \pm 11.63$ & $18.07 \pm 6.14$ & $<0.001$ \\
Actinobacteria & $17.39 \pm 3.52$ & $16.01 \pm 3.73$ & $>0.05$ \\
Bacteroidetes & $6.24 \pm 2.68$ & $6.99 \pm 2.85$ & $>0.05$ \\
Acidobacteria & $4.58 \pm 0.95$ & $5.05 \pm 2.05$ & $>0.05$ \\
Planctomycetes & $3.04 \pm 0.84$ & $3.20 \pm 2.10$ & $>0.05$ \\
Other & $2.96 \pm 1.12$ & $3.58 \pm 1.28$ & $>0.05$ \\
Verrucomicrobia & $2.96 \pm 1.39$ & $3.06 \pm 1.20$ & $>0.05$ \\
Chloroflexi & $2.27 \pm 0.59$ & $2.10 \pm 0.70$ & $>0.05$ \\
Cyanobacteria/Chloroplast & $1.03 \pm 0.66$ & $0.60 \pm 0.41$ & $>0.05$ \\
Nitrospirae & $0.67 \pm 0.39$ & $0.66 \pm 0.55$ & $>0.05$ \\
\hline
\end{tabular}

and 1.50\%), respectively. Notably, Corynebacterium (4.02\%), Pediococcus (3.50\%), and Macrococcus (2.48\%) abundances in the Hoengseong group were significantly different from the Pyeongchang group and constituted members of the dominant bacterial population. The abundances of the genera Acinetobacter (3.74\%), Enhydrobacter (2.54\%), and Chryseobacterium (1.43\%) were also dominant in the Pyeongchang group. In contrast, the Massilia, Enhydrobacter, and Acinetobacter genera were significantly higher in the Pyeongchang group than in the Hoengseong group.

\section{Comparison of raw milk microbiota in the Hoengseong} and Pyeongchang groups

Differences in the microbiota between the two groups were analyzed with beta diversity calculations using the weighted UniFrac to plot PCoA (Figure 2). The analysis showed a slight difference

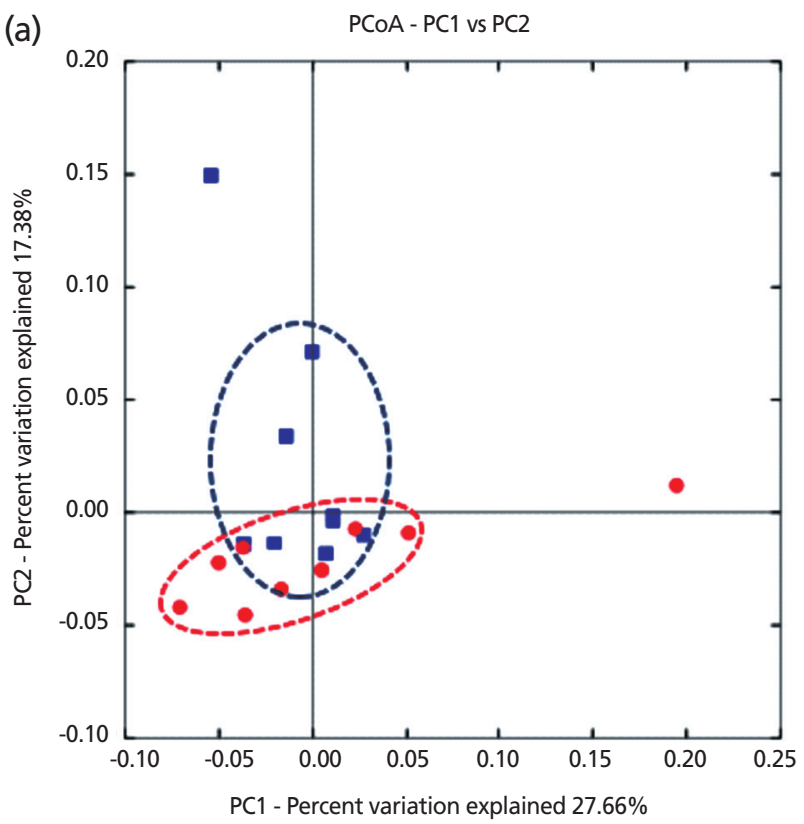

Table 4. The average percentage of predominant bacteria at the genus level between the Hoengseong and Pyeongchang groups

\begin{tabular}{lccc}
\hline \multirow{2}{*}{ Genus level } & \multicolumn{2}{c}{ Relative count (\%) } & \\
\cline { 2 - 3 } & Hoengseong & Pyeongchang & p value \\
\hline Massilia & $6.66 \pm 2.81$ & $9.17 \pm 4.46$ & $<0.001$ \\
Bacillus & $5.14 \pm 2.05$ & $5.29 \pm 2.87$ & $>0.05$ \\
Corynebacterium & $4.02 \pm 3.67$ & $1.47 \pm 0.90$ & $<0.001$ \\
Pediococcus & $3.50 \pm 8.94$ & $0.03 \pm 0.04$ & $<0.001$ \\
Other & $2.96 \pm 1.12$ & $3.58 \pm 1.28$ & $>0.05$ \\
Macrococcus & $2.48 \pm 6.59$ & $1.18 \pm 2.85$ & $<0.01$ \\
Staphylococcus & $1.92 \pm 2.70$ & $2.61 \pm 4.28$ & $>0.05$ \\
Arthrobacter & $1.75 \pm 0.95$ & $2.02 \pm 1.07$ & $>0.05$ \\
Streptococcus & $1.71 \pm 2.41$ & $2.52 \pm 5.82$ & $>0.05$ \\
Burkholderia & $1.66 \pm 0.88$ & $1.50 \pm 1.02$ & $>0.05$ \\
Lactococcus & $1.32 \pm 1.94$ & $0.69 \pm 1.07$ & $>0.05$ \\
Enterococcus & $1.18 \pm 1.32$ & $0.30 \pm 0.50$ & $>0.05$ \\
Bifidobacterium & $1.11 \pm 1.75$ & $0.21 \pm 0.22$ & $>0.05$ \\
Lactobacillus & $0.93 \pm 0.75$ & $0.68 \pm 0.91$ & $>0.05$ \\
Chryseobacterium & $0.80 \pm 1.17$ & $1.43 \pm 3.56$ & $>0.05$ \\
Nitrospira & $0.67 \pm 0.39$ & $0.61 \pm 0.45$ & $>0.05$ \\
Bacteroides & $0.63 \pm 0.50$ & $0.66 \pm 1.00$ & $>0.05$ \\
Clostridium sensu stricto & $0.61 \pm 0.38$ & $0.31 \pm 0.29$ & $>0.05$ \\
Enhydrobacter & $0.60 \pm 0.73$ & $2.54 \pm 4.82$ & $<0.001$ \\
Acinetobacter & $0.36 \pm 0.33$ & $3.74 \pm 5.61$ & $<0.001$ \\
\hline
\end{tabular}

between the Hoengseong and Pyeongchang groups. The separation of the two groups within the PCoA plots is most notable by viewing the PC2 and PC 3 axes, while the PC1 and PC2 axes demonstrated that the trend of all the samples showed similar bacterial communities in the Hoengseong and Pyeongchang samples. According to the PCoA plot with the PC1 and PC2 axes, the microbiome of the Pyeongchang cluster had one outlier and

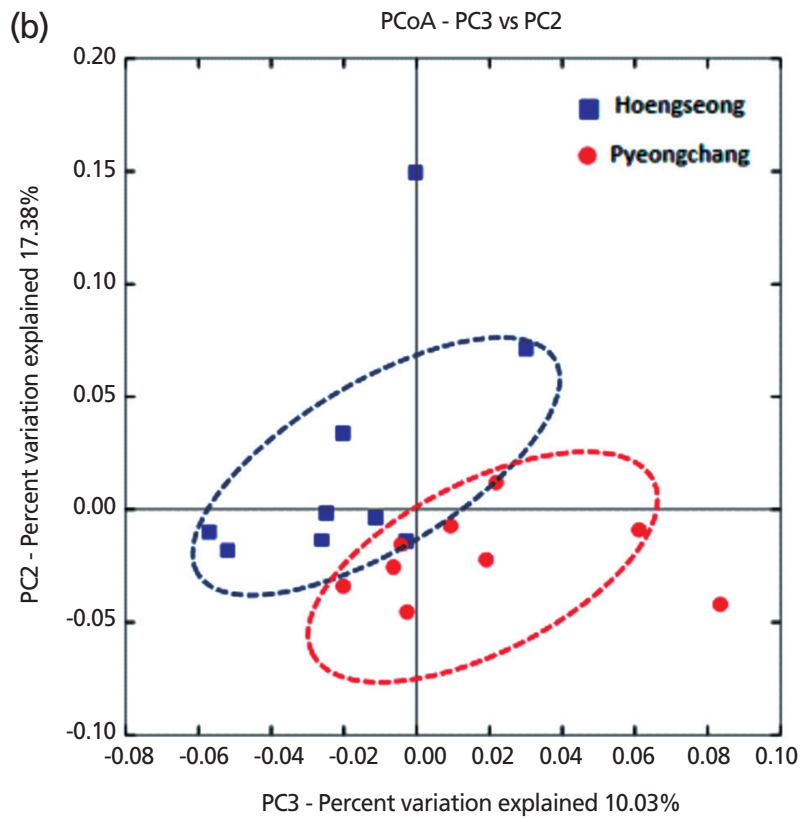

Figure 2. PCoA analysis of the weighted UniFrac distance between Hoengseong and Pyeongchang of bacterial communities. The first, second and third coordinate (PC1, PC2, PC3) explained $27.66 \%, 17.38 \%$, and $10.03 \%$ of the variation, respectively. PCOA, principal coordinate analysis. 
was located in the bottom quadrant, with the exception of 19 samples, and the distribution of the Hoengseong group without samples 04 and 05 samples was located very close to the Pyeongchang group. In addition, an analysis of the weighted UniFrac distances by the unweighted pair-group method using average linkage (UPGMA) showed a slight difference between the two groups (Figure 3). These data demonstrated that the raw milk microbiota of samples $01,06,07$, and 10 from Hoengseong was strongly similarity and that the distance of samples 02,12 , and 14 is the closest between each cluster.

\section{DISCUSSION}

Climatic conditions such as temperature and humidity can affect animal performance and livestock production [13]. The yield of milk production is also related to temperature and humidity factors that are usually used to indicate the level of heat stress [14]. Gangwon-do is one of the coldest regions over the course of the year in Korea. We collected 18 raw milk samples from Hoengseong and Pyeongchang counties of Gangwon-do province in autumn. In this period, Gangwon-do has an average temperature of $12.7^{\circ} \mathrm{C}$ and a humidity of $70.8 \%$ [15]. Interestingly, Piao and Baik [15] suggested that the quality grade, yield grade, and marbling score of Korea cattle steer carcasses are generally best in autumn. Therefore, we assumed that our raw milk samples would have good nutrient composition. If the growth of some bacteria that can affect the milk quality such as psychrotrophic bacteria could be successfully controlled in raw milk, the production of safe and high quality dairy products will be possible.

Although the bacterial population within each milk sample was highly diverse and heterogeneous, the core bacteria could be detected by PCoA of the weighted UniFrac distance metric and relative abundances of specific taxa. This finding coincided with previous observations of raw milk [11,16,17]. Regardless of the sample to sample variations and regional differences, consistent taxa were represented by 4 phyla with the phyla Proteobacteria, Firmicutes, Actinobacteria, and Bacteroidetes which are especially dominant. This result was similarly found in culture-based studies and many other countries [2,18-20].

Typically, it has been reported that raw cow milk primarily contained a lactic acid bacteria (LAB) population including Lactococcus, Streptococcus, Lactobacillus, Pediococcus, Leuconostoc, and Enterococcus spp. by the analysis with either culture techniques or next generation sequencing (NGS) technologies $[2,16,21,22]$. A number of psychrotrophic microorganisms including Pseudomonas, Acinetobacter, and Aeromonas spp. were also detected and their proportions were significant in raw milk. In this study, assays of the milk microbiota of Hoengseong samples primarily

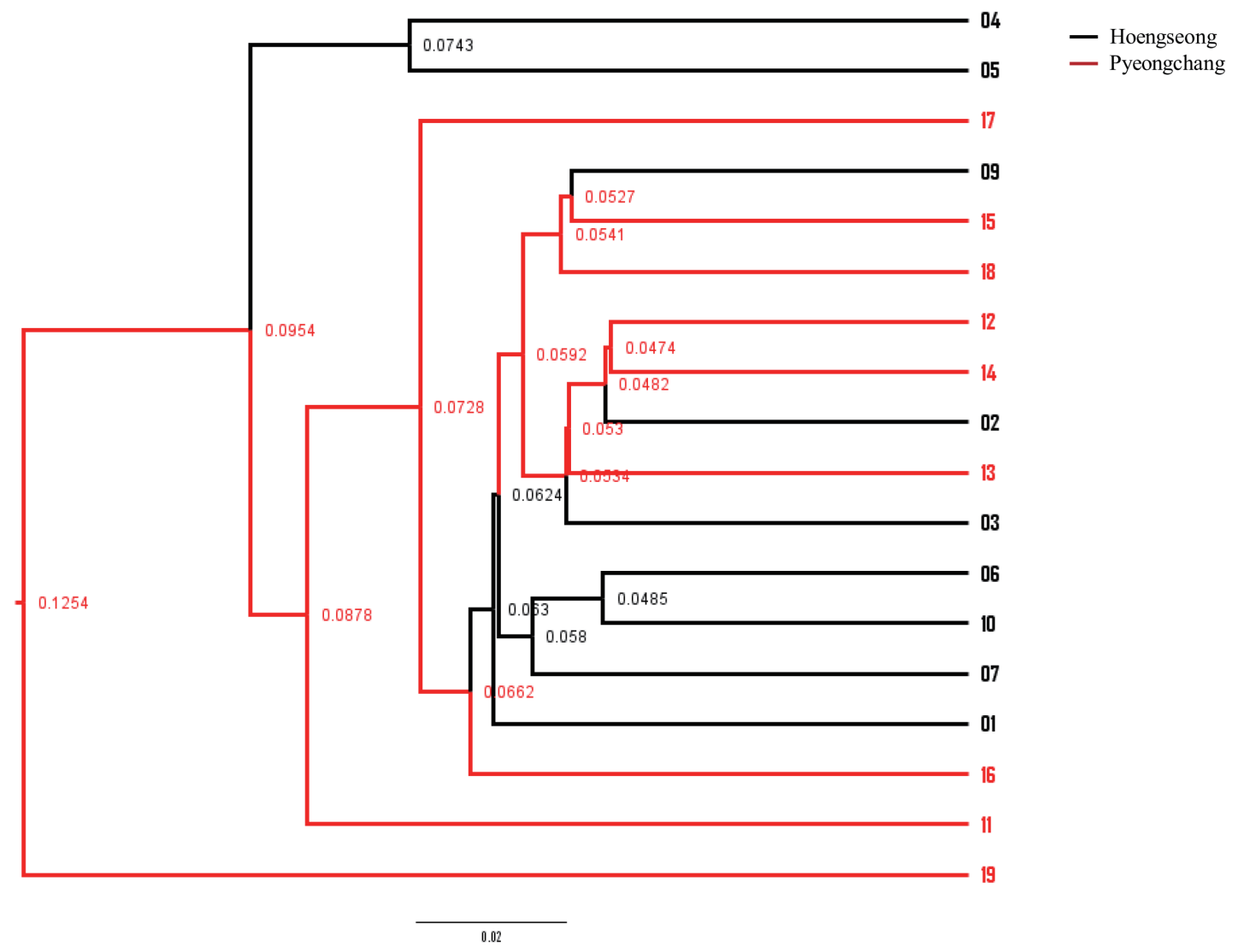

Figure 3. Unweighted pair-group method with arithmetic mean (UPGMA) cluster dendrogram of weighted UniFrac distances from raw milk microbiota of Hoengseong and Pyeongchang. 
contained the abundant LAB (8.64\%), Massilia (6.66\%), Bacillus (5.14\%), Corynebacterium (4.02\%), Macrococcus (2.48\%), and Staphylococcus (1.92\%). In contrast, the milk microbiota of Pyeongchang samples primarily contained Massilia (9.17\%), Bacillus (5.29\%), LAB (4.22\%), Acinetobacter (3.74\%), Staphylococcus (2.61\%), and Enhydrobacter (2.54\%). Based on the NGS analysis, we found that the Proteobacteria, Firmicutes, and Actinobacteria were the predominant phylum in raw milk from Gangwon-do of South Korea but interestingly, the major genera in milk samples were different between Hoengseong and Pyeongchang. Notably, Bacillus, Pediococcus, Berkholderia, Macrococcus, Lactococcus, and Enterococcus appearances in the Hoengseong group were higher than from Pyeongchang group. These genera usually are originated from soil, teat and silage [2]. Therefore, we assumed that these differences can be caused by the equipment and environmental management and the quality of raw milk from Pyeongchang is higher than that of Hoengseong.

Recently, the high-throughput DNA sequencing platform has been utilized to examine the bacterial population in raw milk, and could provide a valuable information for application to commercial products. In Denmark, Masoud et al [21] reported that raw bovine milk contained a significant amount of lactic acid bacteria such as Streptococcus thermophiles and Lactococcus lactis representing $43.7 \%$ and $19 \%$ of the overall bacterial community, respectively. Other bacterial genera including Acinetobacter, Aeromonas, Brevibacterium, Corynebacterium, Lactobacillus, Pseudomonas, and Staphylococcus were also founded [21]. In USA, Kable et al [11] reported on the seasonal microbiota of raw bovine milk in tanker trucks and on the outcomes of storage at commercial processing facilities. A core milk microbiome definitely consisted of Streptococcus (6.5\%), Staphylococcus (5.4\%), and unidentified members of order Clostridiales (6.3\%). Also, they checked that the number of total bacteria estimated by quantitative polymerase chain reaction (qPCR) was approximately 100100,000 cells $/ \mathrm{mL}$. In this study, we confirmed that the number of total bacteria are similar (Table 1). In addition, they revealed a highly diverse microbiota including different pathogens depended on the season and storage facility [11].

The quality and safety of milk is an important issue in the dairy industry. Recently, numerous studies have evaluated the relationship between the milk microbiota and milk quality $[9$, $14,23]$. Milk is stored and transferred in a refrigeration system that can effectively control the growth of most bacteria while at the same time providing a selective advantage for the growth of psychrotrophic microorganisms. These psychrotrophic bacteria come from a variety of sources including the milking equipment, the teat apex, feed, soil, water, and other environments. For example, the bovine teat surface could contain a high diversity of bacterial phyla such as Firmicutes (76\%), Proteobacteria (17.8\%), Actinobacteria (4.9\%), and Bacteroides (1.3\%) [24]. Acinetobacter species are dominant soil bacterial taxa [25]. Thus, it is possible that increases of Actinobacteria population in the soil could be transferred to the cow teat and into the milk.

In conclusion, we confirmed the raw milk microbial communities from the Hoengseong and Pyeongchang counties in Gangwon-do of South Korea. These results could provide the crucial information for identifying and characterizing the microbiota in raw milk to ensure the production of high quality dairy products.

\section{CONFLICT OF INTEREST}

We certify that there is no conflict of interest with any financial organization regarding the material discussed in the manuscript.

\section{ACKNOWLEDGMENTS}

This research was conducted by the Korea Institute of Planning and Evaluation for Technology in Food, Agriculture, Forestry and Fisheries (IPET) through the Agri-Bio industry Technology Development Program, funded by the Ministry of Agriculture, Food and Rural Affairs (MAFRA) (316005-5 and 115084-2). In Seon Kim was supported by the BK21 Plus program.

\section{REFERENCES}

1. von Neubeck M, Baur C, Krewinkel M, et al. Biodiversity of refrigerated raw milk microbiota and their enzymatic spoilage potential. Int J Food Microbiol 2015;211:57-65.

2. Quigley L, O'Sullivan O, Stanton C, et al. The complex microbiota of raw milk. FEMS Microbiol Rev 2013;37:664-98.

3. Wouters JTM, Ayad EHE, Hugenholtz J, Smit G. Microbes from raw milk for fermented dairy products. Int Dairy J 2002;12:91-109.

4. Debarry J, Garn H, Hanuszkiewicz A, et al. Acinetobacter lwoffii and Lactococcus lactis strains isolated from farm cowsheds possess strong allergy-protective properties. J Allergy Clin Immunol 2007;119: 1514-21.

5. Hantsis-Zacharov E, Halpern M. Chryseobacterium haifense sp. nov., a psychrotolerant bacterium isolated from raw milk. Int J Syst Evol Microbiol 2007;57:2344-8.

6. Deeth HC. Lipoprotein lipase and lipolysis in milk. Int Dairy J 2006; 16:555-62.

7. de Oliveira GB, Favarin L, Luchese RH, Mclntosh D. Psychrotrophic bacteria in milk: How much do we really know? Braz J Microbiol 2015:46:313-21.

8. 2016 Annual Report Dairy and Products Annual Korea. Seoul, Korea: USDA Foreign Agricultural Service; 2016.

9. Rodrigues MX, Lima SF, Canniatti-Brazaca SG, Bicalho RC. The microbiome of bulk tank milk: Characterization and associations with somatic cell count and bacterial count. J Dairy Sci 2017;100: 2536-52.

10. O'Connell A, Kelly AL, Tobin J, Ruegg PL, Gleeson D. The effect of storage conditions on the composition and functional properties of blended bulk tank milk. J Dairy Sci 2017;100:991-1003. 
11. Kable ME, Srisengfa Y, Laird M, et al. The core and seasonal microbiota of raw bovine milk in tanker trucks and the impact of transfer to a milk processing facility. MBio 2016;7:e00836-16.

12. Caporaso JG, Jesse Stombaugh JK, Bittinger K, et al. QIIME allows analysis of high-throughput community sequencing data. Nat Methods 2010;7:335-6.

13. Birkelo CP, Johnson DE, Phetteplace HP. Maintenance requirements of beef cattle as affected by season on different planes of nutrition. J Anim Sci 1991;69:1214-22.

14. Bohmanova J, Misztal I, Cole JB. Temperature-humidity indices as indicators of milk production losses due to heat stress. J Dairy Sci 2007;90:1947-56.

15. Piao MY, Baik M. Seasonal variation in carcass characteristics of korean cattle steers. Asian-Australas J Anim Sci 2015;28:442-50.

16. Delbes C, Ali-Mandjee L, Montel MC. Monitoring bacterial communities in raw milk and cheese by culture-dependent and -independent 16S rRNA gene-based analyses. Appl Environ Microbiol 2007; 73:1882-91.

17. Liu W, Zheng Y, Kwok LY, et al. High-throughput sequencing for the detection of the bacterial and fungal diversity in Mongolian naturally fermented cow's milk in Russia. BMC Microbiol 2015;15:45.

18. Zhang R, Huo W, Zhu W, Mao S. Characterization of bacterial community of raw milk from dairy cows during subacute ruminal acidosis challenge by high-throughput sequencing. J Sci Food Agric 2015;95: 1072-9.
19. Doyle CJ, Gleeson D, O'Toole PW, Cotter PD. Impacts of seasonal housing and teat preparation on raw milk microbiota: a high-throughput sequencing study. Appl Environ Microbiol 2017;83:e02694-16.

20. Frétin M, Ferlay A, Verdier-Metz I, et al. The effects of low-input grazing systems and milk pasteurisation on the chemical composition, microbial communities, and sensory properties of uncooked pressed cheeses. Int Dairy J 2017;64:56-67.

21. Masoud W, Vogensen FK, Lillevang S, et al. The fate of indigenous microbiota, starter cultures, Escherichia coli, Listeria innocua and Staphylococcus aureus in Danish raw milk and cheeses determined by pyrosequencing and quantitative real time (qRT)-PCR. Int J Food Microbiol 2012;153:192-202.

22. Raats D, Offek M, Minz D, Halpern M. Molecular analysis of bacterial communities in raw cow milk and the impact of refrigeration on its structure and dynamics. Food Microbiol 2011;28:465-71.

23. Ribeiro Junior JC, Beloti V, Massi FP, Fungaro MHP. Thermoduric psychrotrophic proteolytic microbiota from refrigerated raw milk. Semi Ciênc Agrár 2017;38:267-72.

24. Verdier-Metz I, Gagne G, Bornes S, et al. Cow teat skin, a potential source of diverse microbial populations for cheese production. Appl Environ Microbiol 2012;78:326-33.

25. Piao Z, Yang L, Zhao L, Yin S. Actinobacterial community structure in soils receiving long-term organic and inorganic amendments. Appl Environ Microbiol 2008;74:526-30. 